\title{
Utilidad de la espirometría en preescolares de 4 y 5 años
}

\author{
JURY HERNÁNDEZ C.*, IGNACIO SÁNCHEZ D.**, \\ DANIELLA ARANDA M.***, EUGENIA CAMPOS M.****, NILS L. HOLMGREN P.**, \\ PABLO BERTRAND N.** y SOLANGE CAUSSADE L.**
}

\section{Utility of spirometry in 4 to 5 -years old preschool patients}

Background; Spirometry is the most frequently used pulmonary function test. Previous studies have demonstrated the feasibility of carrying out spirometry in preschool patients. Aim: To describe spirometric values obtained in preschool patients studied in a pediatric pulmonary function laboratory. Patients and methods: We reviewed the spirometries performed -from 1996 to 2004- in 180 preschool patients (4- to 6- years old). Records of volume/time and flow/volume curves of spirometries were obtained with a Schiller SP100 spirometer. Variability of baseline measurements of forced vital capacity $(C V F)$ and forced expiratory volume in one second $\left(F E V_{I}\right)$ was analyzed. Results: The average age of the 180 school patients was 5.4 (SD: \pm 0.39$)$ years and 53\% were male. Either diagnosis or suspicious of asthma was the indication for performing spirometry in 172 (90\%) of these children. One, two and three acceptable spirometric maneuvers were registered in 18 (10\%), $99(55 \%)$ and $63(35 \%)$ patients respectively. Expiratory time was $<3 s$ in 24.4\% (n=43), 3 to $6 \mathrm{~s}$ in $66 \%(n=116)$, and $>6 s$ in 9.6\% (n=17) of patients. Expiratory time was larger in children >5compared to $<5$ - years old $(4.1 \pm 1.3 \mathrm{~s}$ versus $3.1 \pm 0.49 \mathrm{~s} ; \mathrm{p}<0.018)$. VEF $F_{1}$ variability in 162 children having 2 or 3 acceptable maneuvers was $<0.1 \mathrm{~L}$ in $67 \%$, $<5 \%$ in $50 \%$ and $<10 \%$ in $80 \%$. Spirometry was normal in $80.5 \%$, obstructive in $19 \%$ and restrictive in one patient. Conclusions: We confirmed that most of the preschool patients are able to perform an adequate spirometric test in terms of acceptability and reproducibility. We suggest to study a normal population of preshool children in order to have normal local data available.

Key words: preschool patients, pulmonary function, normal values.

\section{Resumen}

Introducción: La espirometría es el método más utilizado para evaluar la función pulmonar. Estudios previos han demostrado la factibilidad de realizar estas pruebas en la edad pre-escolar. Objetivos: Describir los valores espirométricos obtenidos en preescolares derivados al laboratorio de función pulmonar. Material y Métodos: Se analizaron en forma retrospectiva las curvas de volumen/tiempo, flujo/volumen, tiempo espiratorio, $C V F$ y $V E F_{1}$, de las espirometrías realizadas entre 1996-2004 en 180 preescolares. Se utilizó un espirómetro Schiller SP100. Se analizó el coeficiente de variación para CVF y VEF . Resultados: Se realizaron espirometrías en 180 menores de 6 años, con edad promedio de 5,4 \pm 0,39 años, 53\% hombres. La principal indicación de la espirometría fue asma 172/180 (90\%). 10\% de los pacientes realizaron 1 maniobra aceptable, 55\% 2 maniobras y 35\% 3 maniobras reproducibles y aceptables. Considerando el tiempo espiratorio, hubo 43 curvas de < $3 \mathrm{~s}$ (24,4\%), 116 de 3 a $6 \mathrm{~s}$ (66\%) y 17 sobre $6 \mathrm{~s}$ (9,6\%). Comparando los grupos menores y mayores de 5 años, se encontró una diferencia significativa solo en el tiempo espiratorio: 3,1 $\pm 0,49 \mathrm{~s}$ en el primer grupo y 4,1 $\pm 0,13$ en el segundo ( $p<0,018)$. Al evaluar

\footnotetext{
Sección Respiratorio, Departamento de Pediatría. Pontificia Universidad Católica de Chile:

* $\quad$ Residente de Enfermedades Respiratorias del niño.

** Sección Respiratorio Pediátrico. Enfermera Laboratorio Respiratorio Pediátrico.

*** Tecnólogo Médico Laboratorio Respiratorio Pediátrico.

**** Enfermera, Laboratorio Respiratorio Pediátrico.
} 
variabilidad de $V E F_{1}$ en 162 pacientes que realizaron 2 ó 3 maniobras aceptables se encontró que un $67 \%$ mostraba variabilidad $<0,1 \mathrm{~L}, 50 \%<5 \%$ y $80 \%<10 \%$. Las espirometrías fueron normales en 145 (80,5\%) preescolares, con alteración obstructiva en 34 (19\%) y con alteración restrictiva en uno $(0,5 \%)$. Conclusiones: La mayoría de los preescolares cumplieron con los criterios de aceptabilidad y reproducibilidad. Se sugiere realizar valores normales en población preescolar sana de nuestro país, con el objeto de poder comparar los datos de pacientes con sospecha de patología respiratoria.

Palabras claves: función pulmonar, valores normales, pre-escolares.

\section{Introducción}

La espirometría es el método de evaluación de la función pulmonar más frecuentemente usado. La confiabilidad de la espirometría depende de la estandarización de su técnica, particularmente en relación a la mantención del esfuerzo espiratorio durante un tiempo determinado y la capacidad de repetir maniobras similares. Los criterios detallados para la obtención de datos y su interpretación han sido publicados por la Sociedad Americana de Tórax (ATS) ${ }^{1}$, Sociedad Europea de Respiratorio ${ }^{2}$, y Sociedad Chilena de Pediatría ${ }^{3}$.

La espirometría es fácil de realizar, de bajo costo, con criterios de aceptabilidad y reproducibilidad reconocidos en adultos y niños mayores, contándose además con valores de referencia, tanto internacionales ${ }^{4,5}$ como nacionales $^{6}$, que hacen posible catalogar sus resultados según los diferentes patrones de alteración. El grupo de edad pre-escolar presentaría dificultad para realizar maniobras espiratorias adecuadas debido a su corta edad. Sin embargo, se han publicado varias experiencias que demuestran lo contrario, usando recomendaciones técnicas y de interpretación ajustadas para este grupo etario ${ }^{7-12}$. Además algunos de estos autores han presentado valores de referencia, lo que permitiría una mayor exactitud para la interpretación de los valores obtenidos ${ }^{9,10}$. El objetivo del presente trabajo fue evaluar la aceptabilidad y reproducibilidad de la espirometría en preescolares.

\section{Pacientes y Métodos}

Se realizó un análisis retrospectivo y descriptivo de las espirometrías de los 180 preescolares derivados para estudio de patología respiratoria al Laboratorio de Función Pulmonar del Servicio de Pediatría de la Pontificia Universidad Católica de Chile, entre 1996 y 2004. Los niños estaban asintomáticos -desde el punto de vista respiratorio durante- las 2 últimas semanas previo a su evaluación, sin uso de medicamentos broncodilatadores de acción corta las últi- mas 8 horas previo al examen, ó 24 horas para los $\beta$ agonistas de acción prolongada. En el caso de uso de corticoides inhalados su suspensión dependía de la indicación del médico tratante y el objetivo del examen. Las condiciones exigidas para la técnica espirométrica fueron las correspondientes a niños en edad escolar.

Se analizaron los datos de aquellos pacientes en los cuales se obtuvieron curvas espirométricas basales de volumen/tiempo, y de flujo/volumen que cumplían criterios de aceptabilidad para niños mayores: tiempo espiratorio de al menos 3 segundos y/o mínimo 1 segundo de meseta (plateau), forma de la curva adecuada: Flujo Espiratorio Máximo adecuado, sin artefactos por tos, inspiraciones repetidas, cierre glótico. Se utilizó un espirómetro Schiller SP-100 (Suiza, 1991). Previo a la realización de la maniobra, personal capacitado incentivó mediante juegos o estímulos la realización de maniobras de espiración forzada. No se utilizó programas computacionales de estimulación o incentivadores. La técnica consistió en tomar aire en forma profunda y realizar una maniobra de espiración forzada inmediata y mantenida, sin pausa inspiratoria previa, en posición de pie y con pinza nasal.

Se consideró el número de maniobras realizadas, tiempo espiratorio de la curva espirométrica basal, valores de Capacidad Vital Forzada (CVF), Volumen Espiratorio Forzado al primer segundo $\left(\mathrm{VEF}_{1}\right)$, relación $\mathrm{VEF}_{1} / \mathrm{CVF}$, flujo espiratorio forzado al $50 \%$ de la $\mathrm{CVF}\left(\mathrm{FEF}_{50}\right)$, flujo espiratorio forzado entre el 25 a $75 \%$ de CVF ( $\left.\mathrm{FEF}_{25-75}\right)$. Se realizó cálculo de variabilidad para los parámetros de $\mathrm{VEF}_{1}$ y $\mathrm{CVF}$ en base al mejor y menor valor obtenido en los casos en que se contaba con 2 ó más maniobras espirométricas aceptables. Los valores analizados corresponden a cifras absolutas, expresadas en litros. Se consideró la variabilidad en términos de porcentaje de $\mathrm{VEF}_{1}$ y CVF en 5 y $10 \%$ para cada uno de ellos, al igual que variación de $<0,1 \mathrm{~L}$ para cada una de las variables antes mencionadas. Se incluyó dentro del análisis el Coeficiente de Variación (CV) para $\mathrm{VEF}_{1}$ y $\mathrm{CVF}$, expresado como un porcentaje de la desviación estándar (DE) y su promedio en forma individual, para aquellos pacientes 
que lograron por lo menos 2 maniobras espirométricas aceptables $\left(\mathrm{CV}=\mathrm{DE} /\right.$ promedio*100 $^{*}$, expresados como promedio en los distintos grupos estudiados y su Error Medio Estándar (EME). Se revisaron los resultados de espirometrías en base a los valores de referencia según Quanjer ${ }^{5}$, incorporados en el equipo. Se consideró un patrón obstructivo si el valor de $\mathrm{VEF}_{1}$ era menor de $80 \%$ del valor predicho, y/o relación $\mathrm{VEF}_{1} / \mathrm{CVF}$ menor de $80 \%$, y/o $\mathrm{FEF}_{50}$ o $\mathrm{FEF}_{25-75}$ menor de $70 \%$ del valor predicho; patrón restrictivo si el valor de CVF era menor a $80 \%$ del valor predicho, con relación $\mathrm{VEF}_{1} / \mathrm{CVF}$ normal. Se consideran porcentajes ya que no se cuenta con tablas de percentiles para este grupo etario.

\section{Análisis Estadístico}

Los datos estadísticos son presentados como promedio y desviación estándar (DE) si tienen una distribución normal, y como mediana y rangos intercuartiles si tienen una distribución no paramétrica. Las proporciones fueron comparados por $\chi^{2}$ test, $t$ test o Mann-Whitney test . Se consideró valor significativo $\mathrm{p}<0,05$ con intervalo de confianza de $95 \%$.

\section{Resultados}

El número de espirometrías analizadas fueron 180 , con edad de 5,4 $\pm 0,39$ años $(X \pm D E)$, mediana de 5,6 años (rango de 4 años 5 meses a 5 años 11 meses). No hubo pacientes de edad menor a 4 años. La distribución de espirometrías por rango etario fue $10 \%$ para el grupo de 4 a 5 años y $90 \%$ para el grupo de 5 a 6 años. La distribución por género fue $53 \%$ masculino y $47 \%$ femenino (Tabla 1). El gran porcentaje de los pacientes fue derivado al laboratorio de función pulmonar con diagnóstico o sospecha de asma 172/180 (90\%) y en otros pacientes se distribuyó en causas misceláneas como tos crónica, fibrosis quística, disquinesia ciliar, etc.

En cuanto al número de maniobras realizadas, 18 pacientes realizaron una maniobra aceptable (10\%), 99 pacientes realizaron 2 maniobras (55\%) y 63 realizaron 3 maniobras (35\%) aceptables. En base a los criterios de aceptabilidad de la maniobra espirométrica considerando el tiempo espiratorio con un tiempo mínimo de 3 segundos y/o con un plateau en la curva volumen/tiempo, encontramos que $43(24,4 \%)$ tuvieron un tiempo menor de 3 segundos, 116 $(66 \%)$ realizaron una maniobra entre 3 a 6 segundos y en $17(9,6 \%)$ niños fue mayor a 6 segundos. Si evaluamos la variabilidad para el parámetro de $\mathrm{VEF}_{1}$ menor de $0,1 \mathrm{~L}, 67 \%$ de los casos cumplían con este criterio; al considerar el mismo parámetro en términos de variabilidad menor de 5\% entre el menor y mayor valor de $\mathrm{VEF}_{1}$ sólo $50 \%$ cumplían con este criterio, aumentando a $80 \%$ cuando se ampliaba a una variación menor de $10 \%$. Cuando se consideró la variable CVF, el porcentaje de la muestra que encuentra variabilidad menor de $0,1 \mathrm{~L}$ fue de $53 \%$. Cuando esta se evaluó en términos de menos de $5 \%$ entre el mayor y menor valor obtenido encontramos que $39 \%$ cumplían este criterio y un $74 \%$ lo hacían cuando se consideraba una variación del $10 \%$ (Tabla 2). Con respecto al coeficiente de variación $(\mathrm{CV})$, tomado del grupo de pacientes que por lo menos realizó 2 a 3 maniobras aceptables, encontramos que su promedio para $\mathrm{VEF}_{1}$ fue $4,2 \%(\mathrm{DE} \pm 3,3)$ y para CVF fue de $4,4 \%(\mathrm{DE} \pm 3,9)$ (Tabla 3).

Tabla 1. Características de la población en estudio

\begin{tabular}{lr}
\hline Características & Valores \\
\hline $\mathrm{n}$ & 180 \\
Sexo $(\mathrm{n})$ masculino $(\%)$ & $94(53 \%)$ \\
Edad, años $(\overline{\mathrm{X}} \pm \mathrm{DE})$ & $5,5 \pm 0,39$ \\
Talla, cm $(\overline{\mathrm{x}} \pm \mathrm{DE})$ & $115 \pm 5$ \\
Peso, kg $(\overline{\mathrm{X}} \pm \mathrm{DE})$ & $23 \pm 4$ \\
Índice de Masa Corporal $\left(\mathrm{kg} / \mathrm{m}^{2}\right)$ & $17 \pm 2$ \\
& \\
Resultados espirométricos $*$ & \\
Normal & $80,5 \%(145)$ \\
Obstructiva & $19 \%(34)$ \\
Restrictiva & $0,5 \%(1)$ \\
\hline
\end{tabular}

* Resultados expresados en porcentajes (n de pacientes)

Tabla 2. Variación en $\mathrm{VEF}_{1}$, CVF y coeficiente de variación en pacientes con 2 ó 3 maniobras espirométricas aceptables

\begin{tabular}{lrrrr}
\hline Criterio & \multicolumn{2}{c}{$\mathbf{V E F}_{\mathbf{1}}$} & \multicolumn{2}{c}{$\mathbf{C V F}$} \\
& $\mathbf{n}$ & $\boldsymbol{\%}$ & $\mathbf{n}$ & $\%$ \\
\hline Variabilidad <0,1 L & 110 & 67 & 86 & 53 \\
Variabilidad < 5\% & 81 & 50 & 63 & 39 \\
Variabilidad < 10\% & 129 & 80 & 120 & 74 \\
$\begin{array}{l}\text { Coeficiente Variación } \\
(\% \pm \text { EME)* }\end{array}$ & $4,6 \pm 4,1$ & $5,2 \pm 4,3$ \\
\hline
\end{tabular}

*EME = Error medio estándar 
Tabla 3. Comparación de las variables espirométricas según grupos etarios: (A) menor de 5 años, (B) mayor de 5 y menor de 6 años

\begin{tabular}{lccccc}
\hline Variable & \multicolumn{2}{c}{ Promedio } & & DE & p \\
\hline Grupo etario & $\mathrm{A}$ & $\mathrm{B}$ & $\mathrm{A}$ & $\mathrm{B}$ & \\
\% Variabilidad $\mathrm{VEF}_{1}$ & 5,81 & 7,02 & 5,43 & 5,8 & 0,44 \\
\% Variabilidad CVF & 7,2 & 7,5 & 3,9 & 6,6 & 0,81 \\
Variabilidad VEF $(\mathrm{ml})$ & 78,6 & 96,6 & 83,7 & 77,1 & 0,47 \\
Variabilidad CVF $(\mathrm{ml})$ & 111 & 148 & 105 & 95 & 0,47 \\
$\mathrm{CV}^{*} \mathrm{VEF}_{1}$ & 4,2 & 4,6 & 4,2 & 4,1 & 0,73 \\
$\mathrm{CV}^{*}$ CVF & 5,2 & 5,2 & 3,0 & 4,4 & 0,99 \\
Tiempo Espiratorio (s) & 3,1 & 4,1 & 0,49 & 0,13 & 0,01 \\
\hline
\end{tabular}

$* \mathrm{CV}=$ Coeficiente de variación

Sólo un paciente tuvo valores de CV sobre $20 \%$ en ambas variables, este fue considerado para los cálculos totales.

Al comparar si existían diferencias significativas entre los dos grupos etáreos estudiados, se encontró que el grupo de edad menor de 5 años tuvo tiempo espiratorio expresado en mediana de 3,1 segundos en comparación a 4,1 segundos del grupo mayor de 5 años $(p=0,018)$. No se encontró diferencia significativa entre el porcentaje de variabilidad y variabilidad en términos de volumen absoluto de $\mathrm{VEF}_{1}$ y CVF. Lo mismo ocurrió en relación a los $\mathrm{CV}$ entre ambos grupos (Tabla 3).

Al evaluar los resultados de las espirometrías realizadas en base a valores de referencia antes mencionado, $145(80,5 \%)$ de la muestra fue normal, 34 (19\%) demostró un patrón obstructivo y sólo $1(0,5 \%)$ un patrón restrictivo (Tabla 1).

\section{Discusión}

Nuestro estudio demostró que el $90 \%$ de los pre-escolares fueron capaces de generar 2 a 3 maniobras espiratorias forzadas, con tiempo espiratorio mayor o igual a un segundo y en $76 \%$ sobre 3 segundos, lo que concuerda con criterios nacionales de pruebas de función pulmonar en pediatría ${ }^{3}$. El análisis de las curvas de flujo/volumen y volumen/tiempo mostró una adecuada aceptabilidad. El porcentaje de pacientes que lograron una variabilidad de $\mathrm{VEF}_{1}$ y $\mathrm{CVF}$ menor de $10 \%$ fueron 80 y $74 \%$ respectivamente, a diferencia de lo expresado en volumen menor de $100 \mathrm{ml}$ para similares parámetros, obteniéndose un 67 y $53 \%$ respectivamente. Los coeficientes de variación fueron suficientemente bajos como para demostrar una baja variabilidad individual, explicado porque nuestra población principalmente estudiada fueron niños mayores de 5 años, en quienes los resultados fueron más homogéneos.

En nuestro medio, la evaluación y manejo de niños pre-escolares con enfermedad pulmonar se realizan fundamentalmente guiados por el criterio clínico. Además de la espirometría se han desarrollado otras técnicas que permiten evaluar la función pulmonar: oscilometría de impulso para medir resistencia de la vía aérea ${ }^{13-15}$, análisis de reactividad bronquial mediante medición de tensión transcutánea de oxígeno y registro de ruidos pulmonares ${ }^{16,17}$.

Para los niños es ocasionalmente difícil conseguir los estándares de aceptabilidad de ATS para la población de adultos ${ }^{5}$, por lo que la falta de consenso en este punto hace que algunos propongan que tiempos espiratorios menores de 1 segundo sean suficientes para lograr vaciar el volumen pulmonar en forma completa. Se han considerado variaciones de $\mathrm{VEF}_{1}$ y CVF menor del $10 \%$ y menor de $150 \mathrm{ml}$, ya que los criterios de ATS de < 5\% y menos de $100 \mathrm{ml}$ son más restrictivos ${ }^{1}$. Kanengiser y cols ${ }^{7}$, evalúan maniobras espiratorias forzadas en niños entre 3 a 5 años de edad, encontrando que $98 \%$ eran capaces de exhalar al menos 1 segundo. Crenesse y cols ${ }^{8}$ encontraron un bajo número de pacientes capaces de realizar tres maniobras forzadas aceptables; un $21 \%$ de niños realizaron un tiempo espiratorio menor de 1 segundo y 73,4\% mos- 
traron variabilidad de CVF menor a 0,1 litro. En nuestro país, Burgos y col $^{12}$, con un buen nivel de cooperación y la realización de un promedio de 9 curvas flujo/volumen, encuentran que la talla es el mejor predictor de variación de los valores espirométricos en niños de 2 a 5 años de edad. En nuestro estudio, el 53\% de los pacientes tuvo variabilidad de CVF menor de 0,1 litro, $75,6 \%$ de ellos realizando un tiempo espiratorio mayor o igual a 3 segundos; esto último se debe a que se realizaron las espirometrías según las normas exigidas para escolares.

Nuestro trabajo tiene las limitaciones de tratarse de un estudio retrospectivo, en el cual se analizaron los valores de espirometría basales solamente, se utilizó el mejor y el menor de los valores de $\mathrm{VEF}_{1}$ y $\mathrm{CVF}$ para el cálculo de variabilidad y coeficiente de variación, lo que lo hace más exigente aún en comparación a otros que incluyen los dos mejores valores de cada variable. Por otra parte, no se evaluaron los resultados obtenidos posterior al uso de broncodilatador, y por último hubo un número reducido de pacientes en el grupo etario menor de 5 años y ninguno en el grupo menor de 4 años, por lo que no se logró cubrir todo el rango de la etapa preescolar.

Nystad y cols ${ }^{18}$ realizaron espirometría incentivada en preescolares, confirmando que existe clara diferencia en reproducibilidad de las variables antes descritas cuando se acepta un 5\% o un $10 \%$, y similares tendencias cuando se evalúa $\mathrm{VEF}_{0,5}, \mathrm{CVF}$ y $\mathrm{PEF}$, al igual que lo encontrado en nuestro estudio. Vilozni y cols ${ }^{19}$ muestran una experiencia similar, a diferencia de Gracchi $\mathrm{y} \operatorname{cols}^{20}$ quienes sólo encuentran ventaja con respecto a obtener mejores valores en Flujo Espiratorio Máximo con el programa de incentivación. En cuanto a los valores de referencia a utilizar en población preescolar, los estudios realizados han demostrado que existe un aumento de las distintas variables con la edad, a medida que el niño crece hay una disminución de la relación $\mathrm{VEF}_{1} / \mathrm{CVF}$ y una buena concordancia al comparar con valores predictivos en base a ecuaciones de regresión de valores de referencia tradicionalmente utilizados ${ }^{3,4,9}$. Cabe destacar en nuestra serie, la mayor proporción de pacientes con el diagnóstico o sospecha de asma, en relación a lo publicado en la literatura $(90 \%$ vs $70 \%$ respectivamente), en esta se agregan pacientes con fibrosis quística, en quienes la evaluación de la función pulmonar es importante en la progresión de la enfermedad ${ }^{7,21}$. Aurora y cols ${ }^{11}$ sugieren algunos criterios para estandarizar el control de calidad de este examen en los pre-escolares: visualización de todas las curvas obtenidas, verificación de un adecuado inicio de la espiración forzada, análisis de $\mathrm{VEF}_{0,5} \mathrm{y} \mathrm{VEF}_{0,75}$, análisis de $\mathrm{VEF}_{1}$ sólo si el tiempo espiratorio es igual o mayor de un segundo, aceptar variabilidad para CVF y $\mathrm{VEF}_{1} \mathrm{de} \pm 10 \%$ y/o $100 \mathrm{ml}$.

En resumen, nuestro estudio permite afirmar que la realización de espirometría es posible y confiable en preescolares, ya que los resultados analizados cumplen con criterios de aceptabilidad y reproducibilidad. Es de la mayor importancia poder contar con estudios nacionales que incorporen valores de referencia en este grupo etario, para poder establecer comparación en los pacientes portadores de patología respiratoria.

\section{Bibliografía}

1.- AMERICAN THORACIC SOCIETY (Medical Section of the American Lung Association) Standardization of spirometry-1994 Update. Am J Resp Crit Care Med 1995; 152: 1107-36.

2.- QUANJER P H, TAMMELING G J, COTES J E, PEDERSEN O F, PESLIN R, YERANULT J C. Lung volumes and forced ventilatory flows. Report Working Party Standardization of Lung Function Tests, European Community for Steel and Coal. Official Statement of the European Respiratory Society. Eur Respir J Suppl 1993; 16: 5s-40s.

3.- LINARES M, SÁNCHEZ I, CORRALES R, DÍAZ A, ESCOBAR A M. Pruebas de función pulmonar en el niño. Rev Chil Pediatr 2000; 71: 228-42.

4.- KNUDSON R J, LEBOWITZ M D, HOLBERG C J. Changes in the normal maximal expiratory flowvolume curve with growth and aging. Am Rev Resp Dis 1983; 127: 725-34.

5.- QUANJER P H, STOCKS J, POLGAR G, WISE M, KARLBERG J, BORSBOON G. Compilation of reference values for lung function measurements in children. Eur Respir J 1989; (Suppl 4): 184S-261S.

6.- GUTIÉRREZ M, RIOSECO F, ROJAS A, CASANOVAD. Determinación de valores espirométricos en una población chilena normal mayor de 5 años, a nivel del mar. Rev Méd Chile 1996; 124: 1295-306.

7.- KANENGISER S, DOZOR A. Forced expiratory maneuvers in children aged 3 to 5 years. Pediatr Pulmonol 1994; 18: 144-9.

8.- CRENESSE D, BERLIOZ M, BOURRIER T, ALBERTINI M. Spirometry in children aged 3 to 5 years : reliability of forced expiratory maneuvers. Pediatr Pulmonol 2001; 32: 56-61

9.- EIGEN H, BIELER H, GRANT D, CHRISTOPH K, TERRILL D, HEILMAN D, et al. Spirometric pulmonary function in healthy preschool children. Am J Respir Crit Care Med 2001; 163: 619-23.

10.- ZAPLETAL A, CHALUPOVA J. Forced expiratory parameters in healthy preschool children (3-6 years of age). Pediatr Pulmonol 2003; 35: 200-7.

11.- AURORA P, STOCK J, OLIVER C, SAUNDERS C, CASTLE R, CHAZIPARASIDIS G, et al. Quality control for spirometry in preschool children with and without lung disease. Am J Respir Crit Care Med 2004; 169: 1152-9. 
12.- BURGOS P, LARA J. Determinación de valores espirométricos en niños preescolares sanos de la comuna de San Ramón. Tesis Licenciatura Kinesiología Universidad de Chile. http://www.cybertesis.cl/tesis/ uchile/2004/burgos_p/doc/burgos_p.pdf

13.- KLUG B, BISGAARD H. Specific airway resistance, interruptor resistance, and respiratory impedance in healthy children aged 2-7 years. Pediatr Pulmonol 1998; 25: 322-31.

14.- MERKUS P, MIJNSBERGEN J, HOP W, DE JONGSTE J. Interrupter resistance in preschool children: measurement characteristics and reference values. Am J Respir Crit Care Med 2001; 163: 1350-5.

15.- LINARES M, CONCHA I, MEYER R. Correlación entre la espirometría y la resistencia y reactancia respiratoria medida por oscilometría en niños asmáticos. Rev Chil Enfer Respir 2002; 18:90-8

16.- SÁNCHEZ I, CLAVERÍA C, ALVAREZ C, LISBOA C. Tensión transcutánea de oxígeno y sibilancias en la evaluación de la reactividad bronquial en niños sanos y asmáticos de 3 a 6 años. Rev Chil Enf Respir 1997;
13: $138-45$.

17.- PASTERKAMP H. Acoustic markers of airway responses during inhalation challenge in children. Pediatr Pulmonol 2004 (suppl); 26: 175-6

18.- NYSTAD W, SAMUELSEN S O, NAFSTAD P, EDVARSEN E, STENSRUD T, et al. Feasibility of measuring function in preschool children. Thorax 2002; 57: 1021-7.

19.- VILOZNI D, BARKER M, JELLOUSCHEK H, HEIMANN G, BLAU H. An interactive computeranimated system (spirogame) facilitates spirometry in preschool children. Am J Respir Crit Care Med 2001; 164: 2200-5.

20.- GRACCHI V, BOEL M, VAN DER LAAG J, VAN DER ENT C K. Spirometry in young children: shouldanimation programs be used during testing ? Eur Respir J 2003; 21: 872-5.

21.- MAROSTICA P, WEIST A, EIGEN H, ANGELICCHIO C, CHRISTOPH K, et al. Spirometry in 3-to 6- years old children with cystic fibrosis. Am J Respir Crit Care Med 2002; 166: 67-71.

\footnotetext{
Correspondencia a:

Dra. Solange Caussade L.

Sección Respiratorio Pediátrico

Pontificia Universidad Católica de Chile

Lira $85,5^{\circ}$ Piso

Santiago, Chile.

E-mail: mcaussa@med.puc.cl
} 